\title{
Secondary EBV-positive Diffuse Large B-cell Lymphoma of Skeletal Muscle from EBV-positive Primary Diffuse Large B-cell Lymphoma of Thyroid Gland: Case Report and Literature Review
}

\author{
J iang X, Sun M and Liu C* \\ Department of Hematology, The Seventh Affiliated \\ Hospital of Yangzhou University, China \\ *Corresponding author: Chunhua Liu, Department \\ of Hematology, J ingjiang People's Hospital, The Seventh \\ Affiliated Hospital of Yangzhou University, China
}

Received: December 26, 2020; Accepted: J anuary 11, 2021; Published: J anuary 18, 2021

\begin{abstract}
Diffuse Large B-Cell Lymphoma (DLBCL) is the most common lymphoid malignancy in adults, which often takes a nonlymph nodes organ as the primary focus. Primary lymphoid malignancy of thyroid gland is not common in clinic, EBV-positive primary diffuse large B-cell lymphoma of thyroid gland is rare in clinic, and its pathogenesis, treatment and prognosis are rarely studied. We reported an 85-year-old female patient with EBV-positive primary diffuse large B-cell lymphoma of thyroid gland, and the disease eventually relapsed in skeletal muscle of the patient. The pathological type after relapse was consistent with that of the primary focus. As far as we know secondary EBV-positive DLBCL of skeletal muscle from EBV-positive primary DLBCL of thyroid gland. For elderly patients with multiple adverse prognostic factors, individualized treatment on the premise of ensuring their quality of life may be more important.
\end{abstract}

Keywords: Diffuse large B-cell lymphoma; EBV-positive; Primary lymphoid malignancy of thyroid gland; immunomodulatory drug; CD30 expression

\section{Introduction}

DLBCL is the most common non-Hodgkin lymphoma NonHodgkin's Lymphoma (NHL), which usually involves lymph nodes and/or nonlymph nodes organs. The diseases come on the primary focus of thyroid gland are rare in clinic, and most of them belong to the pathological type of DLBCL. EBV-positive DLBCL is a kind of aggressive B-cell lymphoma associated with EBV infection. In 2016, the World Health Organization (WHO) defined EBV-related DLBCL as EBV-positive DLBCL. EBV-positive DLBCL has poor overall therapeutic effect, and is prone to relapse in lymph nodes and/ or nonlymph nodes organs. Primary thyroid lymphoma tends to be confined to the thyroid gland, and may relapse in other nonlymph nodes organs such as gastrointestinal tract in some cases, but it is extremely rare for the relapse to involve skeletal muscle clinically.

\section{Case Presentation}

An 85-year-old female patient had a history of chronic bronchitis, emphysema, lacunar cerebral infarction, adrenal adenoma, lumbar vertebral compression fracture and cholecystectomy. In March, 2020, the patient saw a doctor because the lump was found on the left neck, which moved up and down with swallowing action, without symptoms such as pain, fever, night sweat and weight loss in Group B. CT examination showed that the left lobe of thyroid mass partially encircled the trachea and extended to the left trachea ersophagus groove and the trachea and esophagus were compressed and pushed. On March $26^{\text {th }}$, the left lobe of thyroid gland was resected. During the operation, it was found that the solid mass on the left lobe of thyroid gland was indistinct from the surrounding tissues, and no obvious enlarged lymph nodes were found in the neck. Postoperative
CT showed that the volume of the right lobe, isthmus and residual left lobe of thyroid gland increased obviously, the density decreased, and the local boundary with tracheal wall and upper esophagus was unclear. Tumor pathology: CD3(-), CD5(-), CD10(-), CD20(+), CD79a(+), CD56(-), CD30(+), Ki67(+)80\%, CD21(-), CD23(-), MUM1(+), PAX-5(+), Cyclin D1(-), BCL-2(-), C-myc(+), BCL-6(+), Vimentin(+), $\operatorname{IgD}(-)$, Ckpan (epithelial cell + ), CD68 (histiocyte + ); In situ hybridization: $\operatorname{EBER}(+)$; adnexal lymphoid hyperplasia (left thyroid), combining with immunohistochemistry, is considered as EBV-positive DLBCL. Bone marrow cell morphology, IgH rearrangement, TCR rearrangement and bone marrow biopsy showed no abnormality. The quantitative analysis of EBV-DNA was $3.12 \times 103$ copies/ml. CMV-DNA was quantitatively negative. Hashimoto thyroiditis can be ruled out by thyroid function examination, because the patient is old, and has poor chemotherapy tolerance combining with multi-system underlying diseases. Ann Arbor-Cotswolds is in IE stage, and there is no B group symptom, so the patient was given R2 chemotherapy for six courses. Specific dose: rituximab $600 \mathrm{mg} \mathrm{d}$, lenalidomide $25 \mathrm{mg} \mathrm{qd} \times 10$ for $10 \mathrm{~d}$. After four courses of chemotherapy, CT showed that the volume of right thyroid lobe, isthmus and residual left thyroid lobe was smaller than before. After the fifth course of treatment, the patient developed a local mass in the left leg without obvious pain or redness. The mass gradually increased and pain appeared after the end of the sixth course of treatment of R2 chemotherapy, and the movement was limited. CT examination showed that (Figure 1) the left shank muscle occupied a space with a size of about $96 \times 57 \times 59 \mathrm{~mm}$, with scattered calcification and unclear boundary. MRI showed that (Figure 2) there was a mixed signal mass behind the tibia and fibula in the middle and lower segment of the 


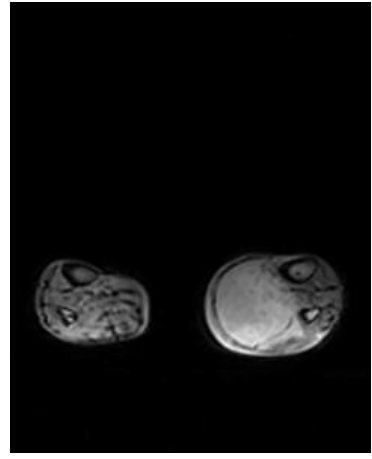

Figure 1: CT: The left shank muscle occupied a space with a size of about $96 \times 57 \times 59 \mathrm{~mm}$, with scattered calcification and unclear boundary.

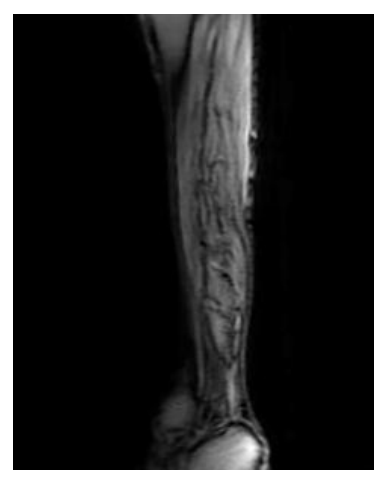

Figure 2: MRI: a mixed signal mass behind the tibia and fibula in the middle and lower segment of the left leg, which was accompanied by calcification and hemorrhage.

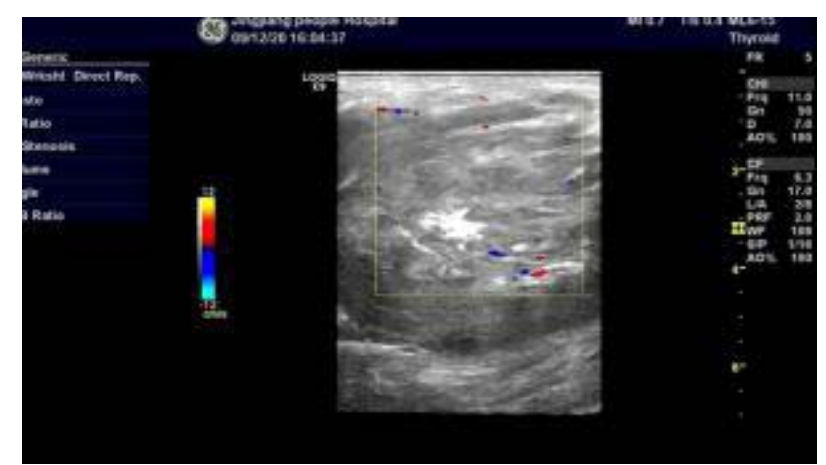

Figure 3: The adjacent leg muscles B-ultrasound: hypoechoic, irregular shape, clear boundary and color blood flow signal were found in the muscle layer of the left leg mass.

left leg, which was accompanied by calcification and hemorrhage, and was indistinct from the adjacent leg muscles B-ultrasound (Figure 3) hypoechoic, irregular shape, clear boundary and color blood flow signal were found in the muscle layer of the left leg mass. The quantitative analysis of EBV-DNA was $7.23 \times 10^{3}$ copies $/ \mathrm{ml}$. CTguided biopsy of left leg mass was performed. Postoperative pathology showed that (Figure 4) the tumor cells were Ckpan(-), Vimentin(+), Ki67(+)80\%, PAX-5(+), MyoD1(-), CD20(+), HMB45(-), Desmin(-), SMA(-), A-103(-), Calponin(-), S100(-), CD56(-), CD3(-), CD10(), MUM1(+), Bcl-2(+)80\%, CD79a(+), C-myc(+)5\%, Bcl-6(+)20\%;

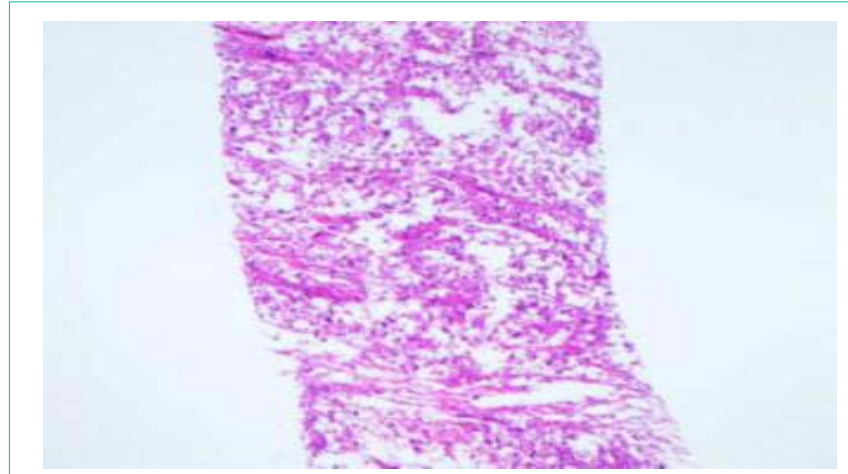

Figure 4: Postoperative pathology showed that Left leg mass biopsy EBVpositive DLBC with necrosis.

dendritic network CD21(-); EBER(+) in situ hybridization; (Left leg mass biopsy) EBV-positive DLBC with necrosis. We think it is the relapse of EBV-positive primary DLBCL of thyroid gland, which involves the left leg skeletal muscle, and the pathological type is consistent with that of primary focus. Patients were treated with $\mathrm{R}$-miniCHOP regimen after relapse. As of press time, a course of treatment with R-miniCHOP regimen has ended, and the mass in the left lower limb of the patient has obviously reduced, and the pain symptoms have basically disappeared, but the chemotherapy-related myelosuppression is obvious, with neutrophil count $<0.5 \times 10^{9} / \mathrm{L}$ and platelet count $<20 \times 10^{9} / \mathrm{L}$.

\section{Discussion}

DLBCL accounts for 30-40 \% of all NHLs in Europe, America and other countries, while it accounts for more than $50 \%$ in China. DLBCL is highly heterogeneous, and its clinical manifestations, disease progression, treatment response and prognosis depend on the pathological type and primary site of lymphoma. Tumor tissue can affect any organ and system, usually in lymph nodes, and 30-40 \% of primary DLBCL is mainly in nonlymph nodes organs. It is common in gastrointestinal tract, skin and soft tissue, bone and genitourinary system, and invades bone marrow, pleura, peritoneum, liver and central nervous system in late stage. DLBCL whose primary focus involves thyroid gland is rare clinically [1-2]. According to the clinical and pathological features of thyroid lymphoma, primary or secondary thyroid lymphoma can be distinguished. It is generally believed that at the first diagnosis, it was found that the tumor only involved the thyroid gland or was dominant in the thyroid gland, and it was confirmed by surgery and fine needle aspiration that the tumor had the pathological characteristics of malignant lymphoma, which could be defined as primary thyroid lymphoma, and there was usually no obvious evidence of lymph node enlargement. Primary thyroid lymphoma Primary Thyroid Lymphoma (PTL) is a rare thyroid malignant tumor, accounting for $0.5-5 \%$ of all thyroid malignant tumors and $3 \%$ of all primary extranodal organ lymphomas on nonlymph nodes organs [3]. 50-80 \% of PTL originated from B cells, among which thyroid diffuse large B-cell lymphoma is the most common pathological type. Typical PTL is common in women aged $60-70$, with a ratio of male to female of about 1:3. The first symptom of male patients is usually 10 years earlier than that of female patients, and the rapid enlargement of painless neck mass is the first symptom. After the mass expands and invades esophagus and trachea, it may be 
accompanied by other symptoms such as dyspnea, dysphagia and hoarseness, and the 5-year survival rate is only 53\% [4]. Hashimoto's Thyroiditis (HT) often occurs in PTL patients, and the incidence of thyroid lymphoma in HT patients is 60-80 times higher than that in the general population, indicating that there is a correlation between this tumor disease and autoimmune diseases [5]. EBER expression was detected in $80.7 \%$ of Hashimoto thyroiditis cases, which indicated that Epstein-Barr virus infection may play a potential role in autoimmune thyroiditis, and the risk of thyroid lymphoma is higher [6]. EBV has a strong ability to induce malignant transformation of lymphocytes, and its carcinogenesis is closely related to the incidence of lymphoma subtypes such as DLBCL. EBV is B-lymphocyte-loving and EBV infection may be the causative factor of human B-cell lymphoma and autoimmune diseases. Long-term chronic inflammatory stimulation in autoimmune state may be the pathogenic basis of malignant tumor development [7], which can explain that more than $90 \%$ of PTL occurs in patients with history of HT. EBVpositive DLBCL has regional tendency, and has different prognosis outcomes in different ethnic backgrounds. The incidence rate in China is higher than that in western countries, and most of them are accompanied by poor prognosis outcomes. The incidence of EBVpositive DLBCL in the elderly is significantly different between the East and the West, and the data reported by different medical centers are different. Generally speaking, EBV-positive DLBCL accounts for $2-3 \%$ of DLBCL in western countries, while it accounts for $8-10 \%$ in Asian countries, and even it has been reported that the proportion can reach $8-15 \%$ [8]. The degradation and inhibition of immune function is considered to be an important mechanism for the occurrence of EBV-positive DLBCL in the elderly. EBER detection in pathological samples is the gold standard for diagnosis of EBVpositive DLBCL. The copy number of EBV-DNA in peripheral blood serum has important application value in diagnosis of EBV-positive DLBCL, dynamic monitoring of disease changes, curative effect evaluation and prognosis judgment. Generally speaking, EBER positive can be used as a significant indicator of poor prognosis of DLBCL, regardless of elderly or young patients. In DLBCL patients, EBV-DNA positive patients have higher relapse rate and worse survival rate than EBV-DNA negative patients, and the progressionfree survival time and total survival time of patients with high serum EBV-DNA load are significantly shortened [9]. According to reports, the median survival time of EBV-positive DLBCL is only 2 years, and the prognosis of patients aged 70 or above and patients with symptoms of group B is even worse. Elderly patients with EBV-positive DLBCL in Asian population have the characteristics of older onset age, later illness, higher frequency of nonlymph nodes organs involvement, higher international prognosis index and higher symptom probability in group B, so the prognosis is significantly worse than that of EBVnegative DLBCL patients [10]. With the wide application of rituximab, the prognosis of DLBCL has been significantly improved, but even under the standard treatment of R-CHOP, $\mathrm{EBER}^{+}$patients still have poor chemotherapy response and poor survival prognosis. The Mutant Allele Tumor Heterogeneity (MATH) score showed that EBV-positive DLBCL showed extensive tumor heterogeneity, which was positively correlated with Ann Arbor stage and multiple nonlymph node focus. The most common mutant genes related to prognosis evaluation in EBV-positive DLBCL include MYC, BCL-2/ BCL-6, TP53, CD30, etc [11]. The expression of these mutant genes has adverse effects on PFS and OS. Clinical reports of EBV-positive primary thyroid DLBCL are rare, accounting for only 4-9 \% of primary thyroid lymphoma cases [4]. There are also regional and ethnic differences in CD30 expression. Evidence shows that the coexpression rate of CD30 in EBV-positive DLBCL in Asian elderly is significantly higher than that in western countries. Patients with coexpression of EBER and CD30 have poor prognosis, and the poor prognosis of EBV-positive DLBCL in Asian population may be related to co-expression of EBER and CD30, not just the expression of EBER [12]. The expression of CD30 and MYC in EBER-positive patients is more common, and has higher Ki-67. Landsburg et al. found that the prognosis of patients with MYC and BCL-6 double expression was worse than that of patients with MYC and BCL-2 double expression, the relapse rate was close to $50 \%$, and OS was only 14.5 months [13]. About $66 \%$ of the relapsed patients with DLBCL have lesions on nonlymph nodes organs, and $50 \%$ of the nonlymph node relapses occur in the previously unaffected sites [14]. The relapse of DLBCL is common in gastrointestinal tract, lung, kidney, spleen and other organs, but the relapse involving skeletal muscle is extremely rare, and the pathogenesis, treatment plan and prognosis have not been systematically expounded. The possible mechanisms of muscle involvement of DLBCL include primary extranodal diseases, local infiltration or disseminated metastasis of adjacent organs including lymph nodes. Under the condition that no adjacent organs are involved, the mechanism of secondary muscle tissue lymphoma is unclear. EBER expression exists in both primary and recurrent focuses of this patient, and EBV can use common regulatory factors to transcribe genes in cells of different lineages, so it is speculated that EBV may play a role in it [8]. According to literature reports, primary thyroid lymphoma tends to be confined in glands. $90 \%$ of thyroid DLBCL is in IE/IIE stage at the time of diagnosis. Most of them have good prognosis and low risk of relapse, and MYC, BCL2/BCL6 expression rarely occurs in thyroid DLBCL. Clinical reports related to EBV-positive primary thyroid DLBCL are very rare. The existing data show that EBV-positive primary thyroid DLBCL is often accompanied by poor prognosis factors such as old age, poor health and CD30 gene mutation, and has poor chemotherapy tolerance, so it is difficult for patients to tolerate the standard regimen and standard dose. Therefore, individualized treatment should be considered for this kind of patients, and the purpose of treatment should be to prolong the survival time on the basis of improving the quality of life. The patient we reported was an elderly woman with multisystem and multiorgan underlying diseases, so we chose R2 regimen for treatment. Lenalidomide, as a new immunomodulatory drug, together with the R2 regimen consisting of rituximab, can enhance the Antibody-Dependent Cell-Mediated Cytotoxicity (ADCC) and reduce the drug resistance of rituximab. They have good synergy and tolerance, avoiding the limitations of monotherapy, and are more suitable for elderly patients who cannot tolerate conventional chemotherapy. However, we did not achieve the expected therapeutic effect, and the relapse on the lower limb skeletal muscle occurred in a short time. After the relapse, we chose R-miniCHOP regimen. Although the clinical symptoms of the patients improved, bone marrow suppression was obvious and lasted for a long time, and repeated fever and hypoproteinemia appeared. We may be the first time to report the case of secondary EBV-positive DLBCL of skeletal muscle from EBV-positive primary DLBCL of thyroid gland. 
Although the patient was in IE stage at the time of diagnosis and had no symptoms of group B, there were many unfavorable prognostic factors such as elderly Asian population, EBV positive, CD30 expression, double expression of C-myc and BCL-6, which could explain the poor treatment effect and extranodal relapse in a short time. The quantitative analysis of EBV-DNA was higher when the disease relapsed than at the initial diagnosis, and the pathological type of recurrent muscle tissue was still EBV-positive DLBCL, which suggested that EBV played an important role. For elderly patients with multiple adverse prognostic factors, individualized treatment on the premise of ensuring their quality of life may be more important.

\section{References}

1. Hatem J, Bogusz A. An unusual case of extranodal diffuse large B-cell lymphoma infiltrating skeletal muscle: a case report and review of the literature. Case Rep Pathol. 2016; 2016: 9104839.

2. Swerdlow SH. World Health Organization, International Agency for Research on Cancer. WHO classification of tumours of haematopoietic and lymphoid tissues. Revised $4^{\text {th }}$ edition. Ed. World Health Organization classification of tumours. Lyon: International Agency for Research on Cancer. 2017.

3. Tavares Bello C, Cassis J, Simoes H, Duarte JS. Nodular lymphocyte predominant Hodgkin lymphoma of the thyroid. Case Rep Endocrinol. 2016; 2016: 8756723.

4. Lam KY, Lo CY, Kwong DL, Lee J, Srivatsava G. Malignant Lymphoma of the Thyroid: A 30-Year Clinicopathologic Experience and an Evaluation of the Presence of Epstein-Barr virus. Am J Clin Pathol. 1999; 112: 263-270.

5. Travaglino A, Pace M, Vavrricchio S, Insabato L, Giordano C, Picardi M, et al. Hashimoto thyroiditis in primary thyroid non-hodgkin lymphoma. Am J Clin Pathol. 2020; 153: 156-164.

6. Janegova A, Janega P, Rychly B, Kuracinova K, Babal P. The role of EpsteinBarr virus infection in the development of autoimmune thyroid diseases. Endokrynol Pol. 2015; 66: 132-136.
7. Graham JP, Arcipowski KM, Bishop GA. Differential B-lymphocyte regulation by CD40 and its viral mimic, latent membrane protein 1. Immunol Rev. 2010; 237: 226-248.

8. Uccini S, Al-Jadiry MF, Scarpino S, Ferraro D, Alsaadawi AR, Ai-Darraji AF et al. "Epstein-Barr virus positive diffuse large B-cell lymphoma in children: A disease reminiscent of Epstein-Barr virus-positive diffuse large B-cell lymphoma of the elderly", Human Pathology. 2015; 46: 716-724.

9. Tisi MC, Cupelli E, Santangelo R, Maiolo E, Alma E, Giachelia M, et al. Whole blood EBV-DNA predicts outcome in diffuse large B-cell lymphoma. Leuk Lymphoma. 2016; 57: 628-634.

10. Park S, Lee J, Ko YH, Han A, Jun HJ, Lee SC, et al. The impact of Epstein Barr virus status on clinical outcome in diffuse large B-cell lymphoma. Blood. 2007; 110: 972-978.

11. Zhou Y, Xu Z, Lin W, Duan Y, Lu C, Liu W, et al. Comprehensive Genomic Profiling of EBV-Positive Diffuse Large B-cell Lymphoma and the Expression and Clinicopathological Correlations of Some Related Genes. Front Oncol. 2019; 9: 683

12. Ok CY, Li L, Xu-Monette ZY, Visco C, Tzankov A, Manyam GC, et al. Prevalence and clinical implications of Epstein-Barr virus infection in de novo diffuse large B-cell lymphoma in Western countries. Clin Cancer Res. 2014; 20: $2338-2349$.

13. Landsburg DJ, Petrich AM, Abramson JS, Sohani AR, Press O, Cassaday R, et al. Impact of oncogene rearrangement patterns on outcomes in patients with double-hit non-Hodgkin lymphoma. Cancer. 2016; 122: 559-564.

14. Adams H, de Klerk J, Fijnheer R, Heggelman BGF, Dubois SV, Nievelstein $\mathrm{RAJ}$, et al. Where does diffuse large B-cell lymphoma relapse? J Comput Assist Tomogr. 2016; 40: 531-536 